\title{
Quantitative and qualitative index for diagnosing low bone density and osteoporosis in post-menopausal women
}

\author{
- Lituania Fialho de Miranda Discipline of Oral Radiology, Department of Stomatology, School of Dentistry, University of \\ São Paulo, São Paulo, SP, Brazil • Emiko Saito Arita Discipline of Oral Radiology, Department of Stomatology, School of \\ Dentistry, University of São Paulo, São Paulo, SP, Brazil • Denise Sabbagh Haddad Discipline of Oral Radiology, Department \\ of Stomatology, School of Dentistry, University of São Paulo, São Paulo, SP, Brazil • Plauto Christopher Aranha Watanabe \\ Discipline of Oral Radiology, Department of Stomatology, School of Dentistry, University of São Paulo, Ribeirão Preto, SP, Brazil
}

ABSTRACT | Objective: The objective of this study was to devise a qualitatively and quantitatively reliable index for screening changes in mandibular bone density based on digital panoramic radiographs. Methods: A total of 252 panoramic radiographs and forearm bone densitometry scans of post-menopausal women were examined. Diagnosis of low density or osteoporosis was confirmed by comparing forearm densitometry diagnostic reports against diagnoses from radiographs. Results: Pearson's tests showed a significant association between assessments by the quantitative and qualitative panoramic index (QQPI) based on panoramic radiography of the mandible and by densitometry ( $<<0.001)$. The Kappa statistic also revealed significant agreement between the two methods of assessment $(\mathrm{p}<0.001)$. Conclusion: These results allowed confirmation of panoramic radiography as a useful method for detecting morphologic age-related changes in the mandible and creation of a single QQPI to aid the diagnosis of mandibular low density/osteoporosis.

DESCRIPTORS | Panoramic Radiography; Bone Mineral Density; Low Density; Osteoporosis.

RESUMO | Índice qualitativo e quantitativo para diagnóstico de baixa densidade óssea e osteoporose em mulheres na pós-menopausa - Objetivo: O objetivo deste estudo foi elaborar um índice qualitativo e quantitativo confiável para rastreio de alterações na densidade óssea mandibular, baseado em radiografia panorâmica digital. Métodos: Foram avaliadas 252 radiografias panorâmicas e densitometrias ósseas de antebraço de mulheres na pós-menopausa. O diagnóstico de baixa densidade ou osteoporose foi confirmado pela comparação da densitometria óssea de antebraço com os diagnósticos realizados por meio de radiografias. Resultados: Os testes de Pearson mostraram uma associação significativa entre avaliações pelo índice panorâmico quantitativo e qualitativo (IPQQ), baseado na radiografia panorâmica da mandíbula, e por densitometria $(\mathrm{p}<\mathrm{o,001)}$. O teste estatístico Kappa demonstrou uma associação significativa entre os dois métodos de avaliação $(\mathrm{p}<\mathrm{o}, 001)$. Conclusão: Esses resultados permitiram a confirmação da radiografia panorâmica como um método útil para a deteç̧̃o de alterações morfológicas relacionadas à idade na mandíbula e criação de um único IPQQ para auxiliar o diagnóstico de baixa densidade/osteoporose mandibular.

DESCRITORES | Radiografia Panorâmica; Densidade Mineral Óssea; Baixa Densidade; Osteoporose.

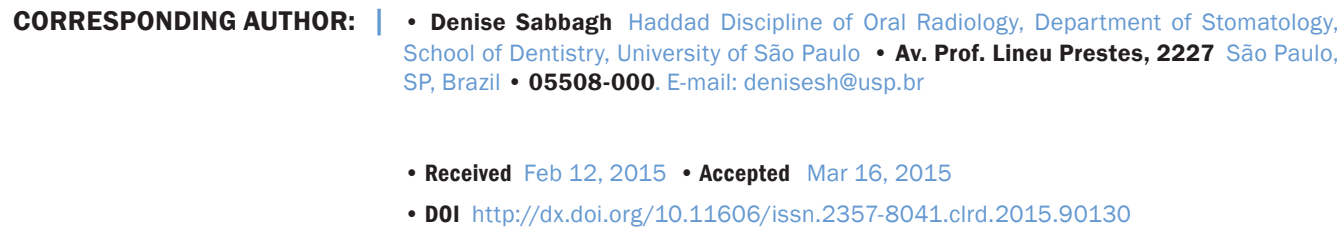

CORRESPONDING AUTHOR: | • Denise Sabbagh Haddad Discipline of Oral Radiology, Department of Stomatology School of Dentistry, University of São Paulo • Av. Prof. Lineu Prestes, 2227 São Paulo, SP, Brazil• 05508-000. E-mail: denisesh@usp.br

- Received Feb 12, 2015 • Accepted Mar 16, 2015

- Dol http://dx.doi.org/10.11606/issn.2357-8041.clrd.2015.90130 


\section{INTRODUCTION}

Osteoporosis is a skeletal disease characterized by impaired bone strength, leading to bone fragility and greater fracture risk. Of the estimated 10 million Americans with osteoporosis, 8 million are women. In these women, bone density may decrease by up to $20 \%$ within 5 to 7 years after menopause, rendering them more susceptible to osteoporosis. ${ }^{1}$

Statistical data for Brazil reveals that, as a result of longer life expectancy among the elderly, the population at risk of developing osteoporosis increased from 7.5 million in 1980 to 15 million in 2000, affecting $35 \%-52 \%$ of women aged 50 years or older. ${ }^{2}$ The risk of fracture is inversely proportional to the individual's bone mineral density (BMD), and is commonly assessed by dual-emission X-ray absorptiometry (DXA). ${ }^{2}$

Recently, methods of assessing radiographic scans for dental use such as panoramic radiography have been suggested as a screening tool for osteoporosis, given the technique's wide availability, simplicity and low cost. In addition, the method has been described as opportune since it would be done on individuals seeking dental care, for whom screening for low BMD is not the main goal. ${ }^{2}$

This may have major implications on public health, as both the chronic asymptomatic progression of osteoporosis (and related clinical problems) and the association between osteoporosis and oral status could be diagnosed earlier with this method. ${ }^{3}$

Although some studies analyzing the effects of low BMD in the maxilla and mandible have found significant results, ${ }^{4}$ others have been less conclusive. ${ }^{5,6}$ Thus, the aim of this study was to devise a qualitatively and quantitatively reliable index for screening changes in mandibular bone density based on digital panoramic radiographs.

\section{MATERIAL AND METHODS Study sample}

The study sample comprised 252 post-menopausal women who were participants of a larger research project whose objective was to correlate BMD with oral status in a group of patients. The present study explored the possibility of devising a single quantitative and qualitative index, exploiting measurements of the mandibular cortex from panoramic radiographs, for identifying low density and osteoporosis cases among a group of postmenopausal women assessed and treated at the clinic of the School of Dentistry of the University of São Paulo (FOUSP), Brazil. After the signing of informed consent forms by participants, panoramic radiographs were taken concomitantly with densitometry scans. Resultant radiographs were assessed by a sole experienced radiologist. Prior to this step, participants filled out a questionnaire collecting information on sociodemographic characteristics and lifestyle for later use in a second phase of this research.

The women who met the study's inclusion criteria were non-smokers, had no systemic diseases that could affect the inflammatory response, immune system or bone metabolism, such as diabetes, hyperparathyroidism, renal osteodystrophy, Paget's disease, fibrous dysplasia, osteomalacia or Pyle's disease, and who were not undergoing treatment for low bone density or osteoporosis.

This project was submitted to the Research Ethics Committee of the University of São Paulo for assessment and was subsequently approved under process number 184/2009.

\section{Bone densitometry scans}

A peripheral dual-energy X-ray absorptiometer (Norland DXA) was used to determine forearm BMD and identify patients at risk of osteoporosis. This device has the following features: it does not 
require X-ray shielding (radiation dose per scan of $0.003 \mathrm{mSv}$ ) or special electrical installation; it can be used on a standard tabletop; it weighs only $27 \mathrm{~kg}$; it can be configured with a laptop computer and portable table for increased mobility; it is suitable for use within a tightly cost-controlled setting; automated daily calibration routine includes precision and accuracy graphs, statistical analysis of phantom scans, including mean value and coefficient of variation; precision and accuracy are equivalent to those of more expensive systems; it produces full, comprehensive and easy-to-read reports; the device offers easy access, positioning accessories improve patient comfort, and the simplicity of the device combined with rigorous quality control ensures proper system operation.

The diagnosis of osteoporosis was confirmed based on densitometric reports. Although the World Health Organization (WHO) defines densitometry scans of the spine and femur as the gold standard for diagnosis, prognosis and monitoring of BMD, this is often not economically viable, and extrapolation of the T-score as a reference measurement has been challenged. Given that forearm bone densitometry is a low-cost method that is easy and fast to apply, safe, non-invasive and portable, involving minimal exposure to radiation and use of physical space; and because the forearm tends not to be influenced by artifacts, deformations or variations and has little soft tissue to hamper assessments of bone mass, this technique rapidly became indicated for investigating low bone mass within communities and rural areas with limited access to health systems, in institutions whose patients have poor ambulation, and for screening axial bone demineralization or guiding treatment plans.

The criteria used to define a diagnosis of osteoporosis were those established by the $\mathrm{WHO}^{7}$ at the Consensus Development Conference (1994), by which low density was defined as a BMD T-score ${ }^{1}$ below $-1 \mathrm{SD}$ and above $-2.5 \mathrm{SD}$. Osteoporosis was defined as a BMD T-score below -2.5 SD. A T-score of $-1 \mathrm{SD}$ or higher was considered indicative of a normal individual.

The value used to determine bone density was Z-score since this compares the patient's bone mass with that of a normal adult of the same age, sex and ethnicity. The person may have a bone mass indicating risk, but this mass may be typical for individuals of their age. In the case of T-score (not considered in this study), patient bone mass is compared with the maximum bone mass of a healthy young adult of the same sex and ethnicity.

\section{Acquisition and assessment of panoramic radiographs}

Digital panoramic radiographs were taken of individuals attending the clinic for diagnosis, treatment and follow-up, using standardized procedures that followed the criteria defined. A Kodak 80ooC Digital Panoramic and Cephalometric System device was employed for radiographs. Energetic parameters $(\mathrm{kVp}, \mathrm{mAs}$ and exposure time) of the device were set according to manufacturer's specifications. The images obtained were identified and stored (on the Phenix online imaging system and individually on data CDs following recommendations provided by the device software).

\section{Determination of the QQPI index (qualitative and quantitative panoramic index)}

The qualitative and quantitative panoramic index was established through a two-stage assessment of the panoramic radiographs, as described below.

\section{Stage One - quantitative analysis}

The quantitative analysis was based on the panoramic mandibular index ${ }^{8}$ (PMI) proposed by Benson et al. in 1991, which determines:

- the ratio of the thickness of the mandibular cortex (measured along a line perpendicular to 
the mandible base at the level of the center of the mental foramen) to the distance between the inferior margin of the base of the mandible and its maximum limit

- normal cortex for thickness greater than or equal to $3 \mathrm{~mm}$ and abnormal cortical density for thickness lower than $3 \mathrm{~mm}$

\section{Stage Two - qualitative analysis}

The same set of radiographs was also classified according to the mandibular cortical index (MCI) of Klemetti et al. $(1994)^{6}$, which qualitatively evaluates the endosteal border of the mandibular cortex, classifying it as $\mathrm{C} 1$ (normal) when smooth and homogeneous, C2 (low density) when exhibiting half-moon defects, and as $\mathrm{C}_{3}$ (osteoporosis) when porous with tapered and thinned cortical width.
In order to determine the QQPI, the two analyses are combined for the same panoramic radiograph, yielding the following classifications:

Q1 = mandibular cortex classified as C1, according to $\mathrm{MCI}$, and $\mathrm{PMI} \geq 3 \mathrm{~mm}$;

Q2 = mandibular cortex classified as $\mathrm{C} 2$, according to $\mathrm{MCI}$, and $\mathrm{PMI}<3 \mathrm{~mm}$;

$\mathrm{Q} 3$ = mandibular cortex classified as $\mathrm{C}_{3}$, according to $\mathrm{MCI}$, and $\mathrm{PMI}<3 \mathrm{~mm}$;

\section{Data analysis procedure}

Results of densitometry and QQPI index (panoramic radiography) were obtained for 252 patients in this study sample. Both tests evaluated patients as normal, with low density or with osteoporosis. Descriptive statistics of absolute (n) and relative (\%) frequencies in the response categories of the tests are given in Table 1.

Table 1 | Absolute and relative frequencies of normal, low density and osteoporosis test categories for panoramic radiographs of the mandible and densitometry scans, and results of tests of association and agreement.

\begin{tabular}{|c|c|c|c|c|c|}
\hline \multirow[b]{2}{*}{ MANDIBLE } & & \multicolumn{3}{|c|}{ DENSITOMETRY } & \multirow[b]{2}{*}{ Total } \\
\hline & & Normal & Low density & Osteoporosis & \\
\hline \multirow[t]{4}{*}{ Normal } & $\mathrm{N}$ & 30 & 29 & 18 & 77 \\
\hline & $\%$ On mandible & $39.00 \%$ & $37.70 \%$ & $23.40 \%$ & \\
\hline & $\%$ On densitometry & $63.80 \%$ & $33.30 \%$ & $15.30 \%$ & $30.60 \%$ \\
\hline & Standardized Residual & 5.5 & 0.7 & -4.9 & \\
\hline \multirow[t]{4}{*}{ Low density } & $\mathrm{n}$ & 14 & 37 & 34 & 85 \\
\hline & $\%$ on MANDIBLE & $16.50 \%$ & $43.50 \%$ & $40.00 \%$ & \\
\hline & $\%$ on DENSITOMETRY & $29.80 \%$ & $42.50 \%$ & $28.80 \%$ & $33.70 \%$ \\
\hline & Standardized Residual & -0.6 & 2.1 & -1.5 & \\
\hline \multirow[t]{4}{*}{ Osteoporosis } & $\mathrm{n}$ & 3 & 21 & 66 & 90 \\
\hline & $\%$ on MANDIBLE & $3.30 \%$ & $23.30 \%$ & $73.30 \%$ & \\
\hline & $\%$ on DENSITOMETRY & $6.40 \%$ & $24.10 \%$ & $55.90 \%$ & $35.70 \%$ \\
\hline & Standardized Residual & -4.7 & -2.8 & 6.3 & \\
\hline \multirow[t]{2}{*}{ Total } & $\mathrm{n}$ & 47 & 87 & 118 & 252 \\
\hline & $\%$ on MANDIBLE & $18.70 \%$ & $34.50 \%$ & $46.80 \%$ & $100.00 \%$ \\
\hline
\end{tabular}

$p$ value (Pearson) $<0.001$

Kappa $=0.284, p$ value $<0.001$ 
The Kappa ${ }^{9}$ method was also applied to assess agreement between the tests. Kappa statistic describes the intensity of agreement between two or more responses of qualitative variables, based on the number of concordant responses. This agreement measure ranges from a maximum value of 1 , indicating total agreement, to a minimum value of zero, or less than $\mathrm{o}$, indicating no agreement. A Kappa value of less than zero (i.e., negative) suggests discordance but does not indicate the degree of discordance. A statistical test is run to assess the significance of the Kappa value. In this case, the hypothesis tested is whether Kappa is equal to $\mathrm{o}$, indicating null agreement, or greater than zero, showing concordance (one-tailed test: $\mathrm{Ho}: \mathrm{K}=\mathrm{O}$; $\mathrm{H} 1 \mathrm{~K}>\mathrm{O}$ ). In the event of rejection of the null hypothesis (Kappa $=0$ ), this indicates the agreement measurement is significantly greater than zero. The assessment as to whether this was satisfactory or not was based on Landis and Koch (1977). SPSS (Statistical Package for the Social Sciences) software version 16 was used for all descriptive statistics and tests. Microsoft Office 2003 Excel was employed for tables and graphs, whereas Word was used for the report.

\section{RESULTS}

Results of Pearson's tests showed a significant association between assessments by panoramic radiography of the mandible and by densitometry ( $\mathrm{p}$ $<$ o.001). Standardized residuals revealed excess of the normal category of the radiograph within the normal category of the densitometry, excess of the low density category of the radiograph within the low density category of the densitometry as well as excess of the osteoporosis category of the radiograph within the osteoporosis category of the densitometry (Figure 2). The Kappa measure also indicated significant agreement between the two tests ( $p<0.001$ ), although the intensity of concordance was considered reasonable $($ Kappa $=0.284)$. The association between results of the panoramic radiography tests of the left and right mandible sides was also significant and, in this case, concordance in responses between the two sides was moderate (Kappa $=0.555 ; \mathrm{p}<0.001)$.

The statistical methods were applied again, this time considering the test responses as absence and presence of osteoporosis, i.e., the normal and low

Table 2 | Absolute and relative frequencies of absent and present osteoporosis test categories for panoramic radiographs of the mandible and densitometry scans, and results of tests of association and agreement.

\begin{tabular}{|c|c|c|c|c|}
\hline \multirow{2}{*}{$\begin{array}{c}\text { Osteoporosis on Mandible } \\
\text { PR }\end{array}$} & & \multicolumn{2}{|c|}{ Osteoporosis on Densitometry Scan } & \multirow[b]{2}{*}{ Total } \\
\hline & & No & Yes & \\
\hline \multirow[t]{4}{*}{ No } & $\mathrm{n}$ & 110 & 52 & 162 \\
\hline & \% Osteoporosis on Mandible PR & $67.90 \%$ & $32.10 \%$ & $100.00 \%$ \\
\hline & \% Osteoporosis on Densitometry Scan & $82.10 \%$ & $44.10 \%$ & $64.30 \%$ \\
\hline & Standardized Residual & 6.3 & -6.3 & \\
\hline \multirow[t]{4}{*}{ Yes } & $\mathrm{n}$ & 24 & 66 & 90 \\
\hline & \% Osteoporosis on Mandible PR & $26.70 \%$ & $73.30 \%$ & $100.00 \%$ \\
\hline & \% Osteoporosis on Densitometry Scan & $17.90 \%$ & $55.90 \%$ & $35.70 \%$ \\
\hline & Standardized Residual & -6.3 & 6.3 & \\
\hline \multirow[t]{2}{*}{ Total } & $\mathrm{n}$ & 134 & 118 & 252 \\
\hline & \% Osteoporosis on Mandible PR & $53.20 \%$ & $46.80 \%$ & $100.00 \%$ \\
\hline
\end{tabular}

$\mathrm{p}$ value (Pearson) $<0.001$

Kappa $=0.386, p$ value $<0.001$ 
density test categories were pooled into a single absence of osteoporosis category (Table 2).

As expected, results of Pearson's tests showed a significant association between the tests by panoramic radiography of the mandible and by densitometry ( $\mathrm{p}<\mathrm{0.001}$ ). The Kappa measure also indicated significant agreement between the two tests ( $\mathrm{p}<$ 0.001), and, although the Kappa value had risen, the intensity of concordance continued to be rated as reasonable $($ Kappa $=0.386)$. The reasonable concordance observed reflects the moderate sensitivity and negative predictive value (NPV) of the test of $56 \%$ and $68 \%$, respectively, whereas the specificity and positive predictive value (PPV) were deemed satisfactory (Table 3).

Table 3 | Values of sensitivity, specificity, PPV and NPV.

\begin{tabular}{c|c|c} 
Sensitivity $(95 \% \mathrm{Cl})$ & $55.90 \%$ & $(46.5 \% ; 65.1 \%)$ \\
\cline { 2 - 3 } Specificity (95\% Cl) & $82.10 \%$ & $(74.5 \% ; 88.2 \%)$ \\
\hline PPV (95\% Cl) & $73.30 \%$ & $(63.0 \% ; 82.1 \%)$ \\
\hline NPV (95\% Cl) & $67.90 \%$ & $(60.1 \% ; 75.0 \%)$ \\
\hline
\end{tabular}

95\%Cl: 95\% Confidence Interval for the proportion

\section{DISCUSSION}

The determination of bone density by panoramic radiograph (PR) can aid the diagnostic screening of patients with low bone density at dental clinics ${ }^{8}$ since this type of scan is routinely performed as part of the dental treatment plan.

Some studies have determined a protocol for assessing mandibular bone density from PRs. ${ }^{6,8,10}$ Benson et al. (1991) sought to develop a radiomorphometric index (PMI) for use with PRs to help identify patients with osteoporosis. These authors determined the shortest distance between the lower edge of the mandible and the inferior border of the mental foramen on panoramic radiographs. The thickness of the cortex was then measured at this same point in the inferior border of the mandible. The index was established and classified as normal for cortex values $\geq 3 \mathrm{~mm}$. This measure was adopted due to the high frequency of the value on assessments performed in individuals of black, hispanic and white ethnicity. A lower PMI in older women of the same ethnic group was also reported, and patients of black ethnicity were found to have a higher PMI, while hispanic and white groups had statistically similar index values.

Klemetti et al. (1994) ${ }^{6}$ assessed 355 post-menopausal women and devised a mandibular cortical index (MCI), which qualitatively evaluates the endosteal border of the mandibular cortex, classifying it as $\mathrm{C} 1$ (normal) when smooth and homogeneous, C2 (low density) when exhibiting half-moon defects, and as $\mathrm{C}_{3}$ (osteoporosis) when porous with tapered and thinned cortex.

Both studies ${ }^{6,8}$ had inherent methodological flaws with respect to the assessment and full usage of the data disclosed by the PRs. In the first instance, the fact that the authors only considered cortical thickness led to exclusion of patients with thick cortices that, nevertheless, had morphological abnormalities. Moreover, the failure to differentiate between low density and osteoporosis rendered the study less specific compared with the present investigation. With the second study, the opposite occurred since the cortical morphology assessment was restricted by not taking into account thickness. Consequently, the diagnoses of patients in both studies lacked precision and efficiency.

The index employed in the present study (QQPI) proposes the use of both methodologies suggested by the cited authors, ${ }^{6,8}$ combining quantitative and qualitative analyses of the mandible and observing both the thickness and aspect of the mandibular cortex in determining density, with three possible diagnostic outcomes: normal (Q1), low density (Q2) and osteoporosis (Q3).

The Q1 classification (normal) is assigned for cases with a mandibular cortex classified as $\mathrm{C} 1$ (by Klemetti) on the MCI (smooth and homogenous) and with a PMI of $\geq 3 \mathrm{~mm}$, indicating patients with 
no substantial changes in the mandibular cortex. The Q2 classification is assigned for cases with a mandibular cortex classified as $\mathrm{C} 2$ (by Klemetti), with the presence of tenuous defects according to the MCI, and a PMI of $<3 \mathrm{~mm}$, indicating patients with cortical changes. Finally, the Q3 classification is assigned for cases with a mandibular cortex classified as $\mathrm{C}_{3}$ (by Klemetti), with a porous cortex according to the MCI, and a PMI of $<3 \mathrm{~mm}$, defining patients exhibiting evident changes indicative of osteoporosis.

However, in order to be considered valid, the proposed index must offer good sensitivity and specificity compared with the results of bone densitometry. In the present study, agreement between the two methods of assessment, rated as reasonable, reflects sensitivity of $56 \%$ (moderate), specificity of $82 \%$ (satisfactory), and NPV of $68 \%$.

According to data from Table 1, of the 77 patients with mandibles considered normal by PR, 47 were considered normal by densitometry; of the
85 patients with mandibles classified as indicative of low density by PR, 87 were confirmed by densitometry as cases of low density, and of the 118 were confirmed by densitometry as cases of osteoporosis. These data show that diagnosis by PR may overestimate the number of normal assessments and underestimate the frequency of osteoporosis cases, perhaps indicating false negatives in the sample. In addition, the near perfect agreement on low density diagnoses indicates that, although this assessment is the most difficult and subjective of the three, it nevertheless showed the highest sensitivity.

According to Figure 1 comparing results of densitometry versus mandible PR, the frequency of outcomes assessed as normal on mandible PRs was greater (63.8\%) than on densitometry scans, and likewise for low density (42.5\%) and osteoporosis (55.9\%). In light of these results, the Kappa measure indicated significant agreement between the two methods of assessment ( $p<0.001)$, although the intensity of concordance was deemed

\section{Frequencies of responses on mandible radiographs within each response on dens itometry scans}

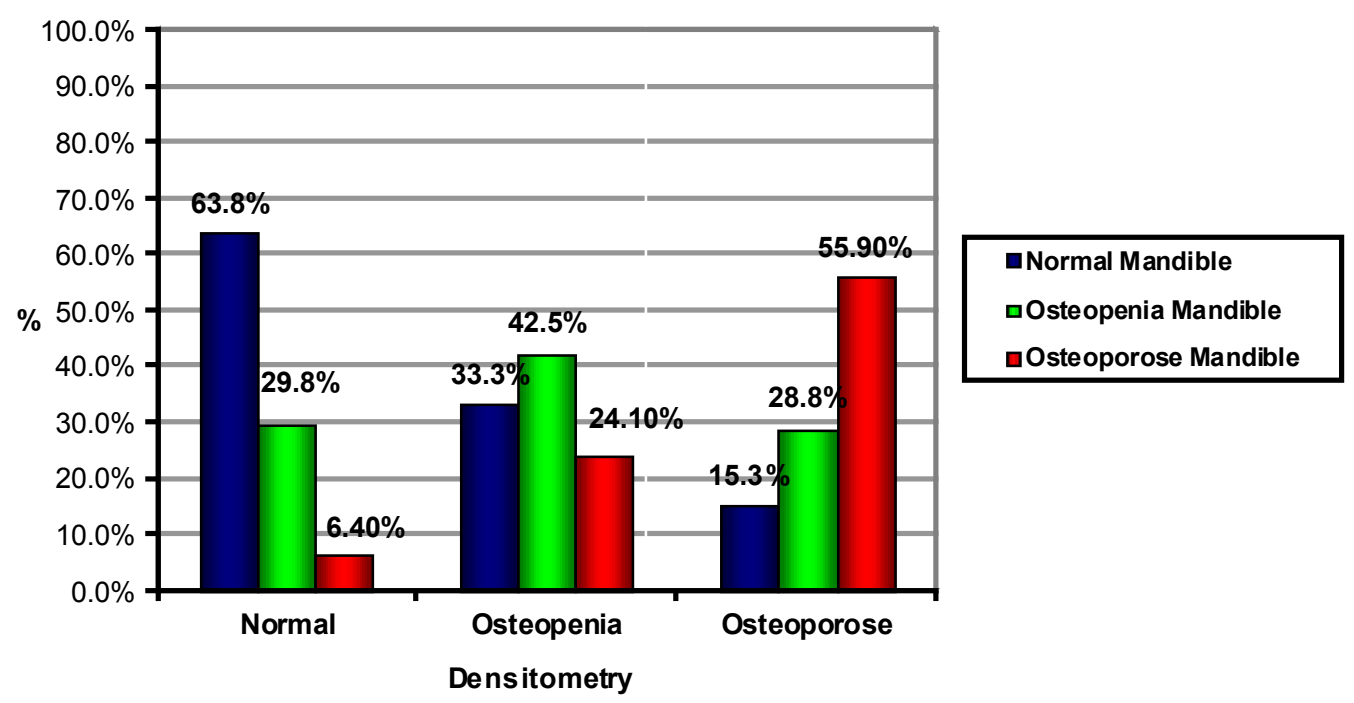

Figure 1 | Distribution of frequencies of responses on panoramic radiographs of the mandible within each response on densitometry scans. 


\section{Frequencies of results of mandible radiographs within categories of densitometry scans}

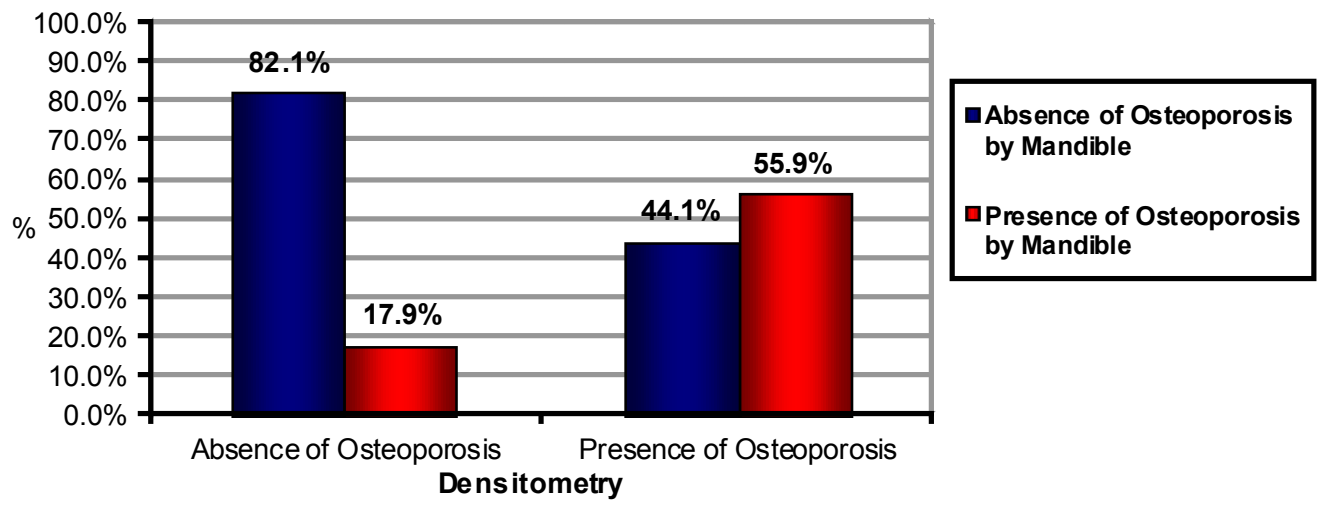

Figure 2 | Distribution of frequencies of responses on panoramic radiographs of the mandible within each response on densitometry scans.

reasonable $($ Kappa $=0.284)$ based on the interpretation suggested by Landis and Koch (1977).

Also in Table 1, of the 47 cases with normal diagnoses on densitometry scans, 30 were assessed as normal on PRs, 14 as low density and 3 as osteoporosis. Of the 87 cases diagnosed with low density on densitometry, 29 were assessed as normal on PRs, 37 as low density and 21 as osteoporosis. Finally, of the 118 cases diagnosed as osteoporosis on densitometry, 18 were assessed as normal on PRs, 34 as low density and 66 as osteoporosis. These data reveal that the image of a normal cortex by PR is easily confounded with the aspect of low density, indicating low specificity of the measure. The results also show that the image disclosing osteoporosis is seldom confounded with a normal diagnosis on PR, indicating high specificity between normal and osteoporosis classifications.

According to data in Table 2, depicting only the presence or absence of osteoporosis, the following results were obtained: for 162 mandibles assessed as non-indicative of osteoporosis, 134 densitometry scans were also negative for osteoporosis, and for
90 mandibles assessed as indicative of osteoporosis, 118 densitometry scans also tested positive for the condition. The Kappa measure also showed significant agreement between the two tests ( $\mathrm{p}<$ o.001), and despite an increase in the Kappa value, the intensity of concordance remained reasonable $($ Kappa $=0.386) .{ }^{11}$

The only way of accurately determining the absolute mandible bone density would be determining the weight of the mandibles after incineration. However, evidence suggests that the shape and thickness of the mandible cortex on panoramic radiographs can be used as a tool for detecting low bone density. In addition, the identification of mandibular cortex erosion by general clinical dentists on panoramic radiographs of post-menopausal women leads to detection of low BMD in $73 \%$ of cases. ${ }^{12}$ In this context, the results of the present study appear promising.

Several limitations of this study should be pointed out. Firstly, radiographs exhibiting a cortical value of $\geq 3 \mathrm{~mm}$ were considered Q1 (normal) even in the presence of morphological abnormalities. This may have led to false negative results for 
diagnoses among individuals with abnormal systemic bone density, perhaps explaining the overestimation of cases classified as having normal cortex. However, this question shall be addressed, together with other potentially interfering variables, in a second phase of the study.

The panoramic mandibular index ${ }^{8}$ proposes the use of a scale in millimeters to measure the cortex traced on acetate slides overlaid onto conventional panoramic radiographs. In the present study, this measurement was performed by computer using Image $J$ software, allowing more accurate measurements than those of the 1991 study. This approach may explain the reasonable Kappa value found in this study compared with the moderate value reported in the 1991 study by Benson et al.

Studies comparing all indexes for the same sample are scarce. In a study comparing PMI, mandibular index (MI) and MCI measured bilaterally on panoramic radiographs of 94 women divided into three groups (normal, low density and osteoporosis), the authors ${ }^{14}$ concluded that cortical thickness and shape reflect the systemic condition of bone mass loss. The findings of the present study are in line with these results.

Some authors recommend MI values of less than $3 \mathrm{~mm}$ as a criterion for referring patients for bone densitometry scans. ${ }^{15}$

The dual-emission X-ray absorptiometry (DXA) scanning technique assesses base levels of osteoporotic bone of the spine, femur and forearm. This method, however, is not used for the mandible. Comparisons can be made with pre-determined reference values for these regions to detect the existence or otherwise of bone mass loss in the body. ${ }^{16}$

Comparison of mandibular BMD with that of other non-gnathic sites commonly used for identifying osteoporosis has been done using dual-emission densitometry. ${ }^{18,19}$ In the first of the two studies, the authors found a strong correlation between
BMD of the mandible body and the forearm, urging further studies to confirm the utility of mandibular body mineral density as an indication of osteoporosis.

Corroborating this study, the use of radiomorphometric indexes to identify changes in mandibular bone density was evaluated by Brazilian authors, ${ }^{2}$ with results supporting that assessment of mandibular cortical bone thickness can be used to confirm the diagnosis of individuals with low BMD.

The QQPI showed significant association and concordance with densitometry results (gold standard) and may be used as a screening test, albeit with care, insofar as a reasonable intensity of concordance was attributable to moderate sensitivity of this test in relation to the gold standard.

The present study highlighted the importance of indicating a specialized scan for patients with changes in mandibular BMD, allowing early management of this systemic condition through prevention and treatment. Thus, it is important that dentists be aware of this index and prepared to interpret its results and work together with other health professionals in assessing risk for osteoporosis.

In conclusion, the results of this study allowed confirmation of digital panoramic radiography as a useful method for detecting morphologic agerelated changes in the mandible and creation of a single quantitative and qualitative panoramic index (QQPI) to aid in the diagnosis of mandibular low density /osteoporosis.

\section{REFERENCES}

1. Jiang X, Schnatz PF. Denosumab: an antifracture therapy for postmenopausal women with osteoporosis. Menopause. 2013 Feb;20(2):117-9.

2. Passos JS, Gomes Filho IS, Sarmento VA, et al. Women with low bone mineral density and dental panoramic radiography. Menopause. 2012 Jun;19(6):704-9. 
3. Kribbs PJ, Chesnut CH, Ott SM, Kilcoyne RF. Relationships between mandibular and skeletal bone in a population of normal women. J Prosthet Dent. 1990 Jan;63(1):86-9.

4. Groen JJ, Menczel J, Shapiro S. Chronic destructive periodontal disease in patients with presenile osteoporosis. J Periodontol. 1968 Jan;39(1):19-23.

5. Elders PJ, Habets LL, Netelenbos JC, van der Linden LW, van der Stelt PF. The relation between periodontitis and systemic bone mass in women between 46 and 55 years of age. J Clin Periodontol. 1992 Aug;19(7):492-6.

6. Klemetti E, Kolmakov S, Kröger H. Pantomography in assessment of the osteoporosis risk group. Scand J Dent Res. 1994 Feb;102(1):68-72.

7. World Health Organization. Assessment of fracture risk and its application to screening for postmenopausal osteoporosis. Report of a WHO Study Group. World Health Organ Tech Rep Ser. 1994;843:1-129.

8. Benson BW, Prihoda TJ, Glass BJ. Variations in adult cortical bone mass as measured by a panoramic mandibular index. Oral Surg Oral Med Oral Pathol. 1991 Mar;71(3):349-356.

9. Fleiss JL. The statistical basis of meta-analysis. Stat Methods Med Res. 1993;2(2):121-145.

10. Taguchi A, Suei Y, Ohtsuka M, Otani K, Tanimoto K, Ohtaki M. Usefulness of panoramic radiography in the diagnosis of postmenopausal osteoporosis in women. Width and morphology of inferior cortex of the mandible. Dentomaxillofac Radiol. 1996 Nov;25(5):263-7.
11. Landis JR, Koch GG. The measurement of observer agreement for categorical data. Biometrics. 1977 Mar;33(1):159-74.

12. Taguchi A, Ohtsuka M, Nakamoto T, Tanimoto K. [Screening for osteoporosis by dental panoramic radiographs]. Clin Calcium. 2006 Feb;16(2):291-7.

13. Taguchi A, Tsuda M, Ohtsuka M, et al. Use of dental panoramic radiographs in identifying younger postmenopausal women with osteoporosis. Osteoporos Int. 2006;17(3):387-94.

14. Kim IH, Mupparapu M. Dental radiographic guidelines: a review. Quintessence Int. 2009 May;40(5):389-98.

15. Devlin H, Karayianni K, Mitsea A, et al. Diagnosing osteoporosis by using dental panoramic radiographs: the OSTEODENT project. Oral Surg Oral Med Oral Pathol Oral Radiol Endod. 2007 Dec;104(6):821-8.

16. Kleerekoper M. Extensive personal experience: the clinical evaluation and management of osteoporosis. J Clin Endocrinol Metab. 1995 Mar;80(3):757-63.

17. Horner K, Allen P, Graham J, et al. The relationship between the OSTEODENT index and hip fracture risk assessment using FRAX. Oral Surg Oral Med Oral Pathol Oral Radiol Endod. 2010 Aug;110(2):243-9.

18. Horner K, Devlin H, Alsop CW, Hodgkinson IM, Adams JE. Mandibular bone mineral density as a predictor of skeletal osteoporosis. Br J Radiol. 1996 Nov;69(827):1019-25.

19. Horner K, Devlin H. The relationship between mandibular bone mineral density and panoramic radiographic measurements. J Dent. 1998 May;26(4):337-43. 